E R R A T A

ODD $\Lambda \Sigma$ PARITY AND THE NATURE OF THE $\pi \Lambda \Sigma$ COUPLING. Y. Nambu and J. J. Sakurai [Phys. Rev. Letters $\underline{6}, 377$ (1961)].

We would like to emphasize that the parity combination

$$
P(\Lambda \Sigma)=-1, P(K \Lambda N)=-1, P(K \Sigma N)=+1
$$

[where $P(\Lambda \Sigma)$ stands for the relative $\Lambda \Sigma$ parity, etc.] has been previously considered by S. Barshay [Phys. Rev. Letters 1 , 97 (1958); Nuclear Phys. 13, 435 (1959)].

\title{
LARGE ANISOTROPIC KNIGHT SHIFTS IN
} INTERMETALLIC COMPOUNDS. R. G. Barnes, W. H. Jones, Jr., and T. P. Graham [Phys. Rev. Letters $\underline{6}, 221$ (1961)].

Our Letter overlooked the fact that Jaccarino, Matthias, Peter, Suhl, and Wernick ${ }^{9}$ had reported quantitative values with respect to the anisotropic Knight shifts of $\mathrm{Al}^{27}$ in certain Laves phase compounds. The last sentence of the second-last paragraph should be replaced by: "On the other hand, as has also been remarked by Jaccarino et al. , ${ }^{9}$ a major part of the large anisotropy in the case of $\mathrm{TmAl}_{2}$ very likely arises from the nonisotropic part of the $s-f$ exchange interaction, the isotropic part of which has been shown ${ }^{9}$ to account for the large Knight shifts in the rareearth-aluminum compounds. The latter authors also reported observing anisotropic shifts in the rare-earth-aluminum Laves phase compounds." 